\title{
Multi-objective robust design optimization of the mechanism in a sewing machine
}

\author{
Nejlaoui Mohamed ${ }^{1}$, Najlawi Bilel ${ }^{1,{ }^{*}}$, Zouhaier Affi $^{1}$, and Lotfi Romdhane ${ }^{2}$ \\ ${ }^{1}$ LGM, ENIM, University of Monastir, Monastir, Tunisia \\ ${ }^{2}$ Department of Mechanical Engineering, American University of Sharjah, Sharjah, UAE
}

Received: 13 June 2016 / Accepted: 4 January 2017

\begin{abstract}
This work deals with the multi-objective robust design optimization of a needle-bar-and-threadtake-up-lever (NBTTL) mechanism used in sewing machines. A multi-objective imperialist competitive algorithm combined with the Monte Carlo method is developed and used for the robust multi-objective optimization of the NBTTL mechanism. This robust optimization minimizes simultaneously the needle jerk, the maximum angular velocity ratio and their standard deviations when the design parameters are known with a given uncertainty. By comparison with Juki 8700, the obtained results showed that the developed algorithm can give a robust design of a sewing machine with the same performance as the those obtained by deterministic methods but less sensitive to the design parameters uncertainties.
\end{abstract}

Keywords: Robust design / sewing machine / uncertainty / imperialist competitive algorithm / Monte Carlo simulation

\section{Introduction}

Many researchers focused on optimizing the designs of sewing machines in order to improve their mechanical performances. Some works, treated the effect of the design parameters (DPs) of the needle-bar-and-thread-take-uplever (NBTTL) mechanism on their performances. Payvandy and Ebrahimi [1] used a mono-objective function with penalty weighting factors to minimize the coupler tracking error and the Jerk of the thread take-up lever. Ebrahimi et al. [2] presented an optimization problem based on the imperialistic competitive algorithm (ICA) for optimizing the needle velocity and the variation of needle acceleration in a link drive mechanism of a sewing machine. Komarec et al. [3] develop a mono-objective optimization of the needle transfer mechanism by means of the software Pro/Engineer Wildfire 4 with the aim to obtain a reduction of needle wear.

It is a common practice in sewing machine design optimization to consider the nominal value of DPs as input variables. However, the sewing machine DP values are usually known with a certain uncertainty. This uncertainty is caused by several factors, i.e., manufacturing errors, geometric errors and material properties. To estimate the effect of DP uncertainty on the performance of a

\footnotetext{
* e-mail: najlawibilelali@gmail.com
}

mechanical system, several methods have been used. In particular, the Monte Carlo simulation (MCS) is a popular and simple tool in estimating the effect of uncertainties on the output of a process [4]. Chandrashekhar and Ganguli [5] developed an optimization method based on fuzzy logic for damage detection in the steel beam having material uncertainty. To evaluate the frequency variations of a beam, they performed a probabilistic analysis using the MCS. Cloupet et al. [6] used the MCS method to estimate the wear variability of rolling contact in industrial machinery caused by uncertain friction coefficient. Motevalli et al. [7] applied the MCS to study the impact of water inflow uncertainty on the performance of both single and multi-reservoir systems.

Other works have studied robust design product where uncertainties of the DPs are considered. Cheng and Li [8] have developed a hybrid differential evolution and sequential quadratic programming method for the robust mechanical structures under uncertain DP. Kalantari et al. [9] have developed a hybrid robust evolutionary algorithm by combining the NSGA-II process with a local search method. This strategy is used to optimize composite structures under an uncertain fibre angle and a lamina thickness. Sun et al. [10] developed a robust optimization algorithm based on successive Taguchi approach for the crashworthiness design of hybrid steelaluminum tailor welded thin-walled structures. Ghanmi et al. [11] studied the robust design optimization of 

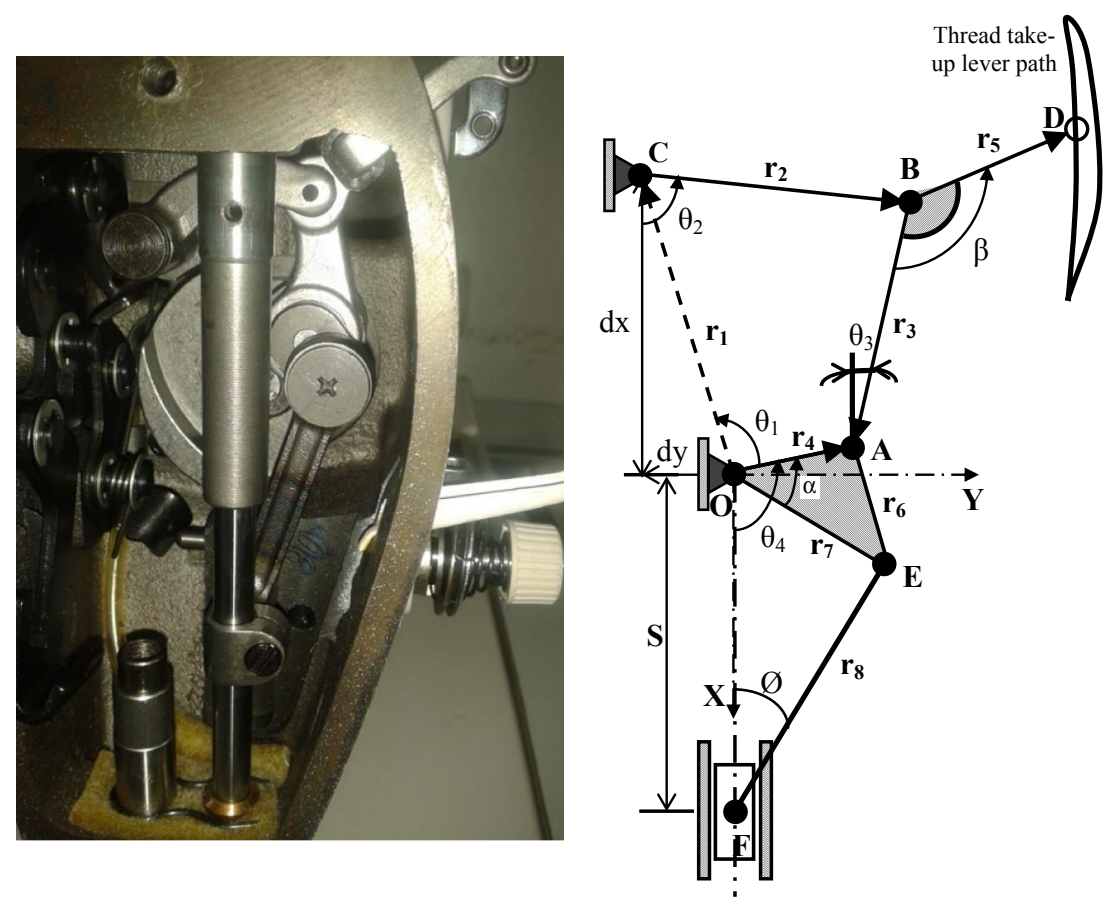

Fig. 1. The NBTTL mechanism.

complex mechanical structures using the genetic algorithm (GA). The robustness, defined by the ratio of the mean value to the standard deviation, is treated as an objective function.

This paper deals with the multi-objective robust design optimization of the NBTTL mechanism used in sewing machines. A combined algorithm based on the multiobjective imperialist competitive algorithm (MOICA) and the MCS is proposed and used. The obtained results are analyzed and compared to the commercialized sewing machine Juki 8700.

The remainder of this paper is organized as follows. In Section 2, the functioning of the NBTTL mechanism is described. Section 3 presents the objective functions to be considered in the NBTTL mechanism design. Section 4 deals with the deterministic multi-objective design optimization. In Section 5, a combined algorithm is developed and used in the multi-objective robust design optimization. Finally some concluding remarks are presented.

\section{The NBTTL mechanism}

Figure 1 presents the NBTTL mechanism of a sewing machine. The thread take-up lever mechanism is the fourbar linkage OABC. OEF represents the slider-crank mechanism in which point $\mathrm{F}$ denotes the needle. The function of the thread take-up lever, in the stitch formation process, is to en-sure appropriate thread feeding. The function of the needle, which is fixed to the needle bar, is to penetrate the fabric. The rotation of the input link $(\mathrm{OA})$ is transformed to the translation of the needle bar through the slider-crank mechanism. The displacement of the needle bar is represented by the distance " $S$ ".

\section{Kinematics of the NBTTL mechanism}

To optimize the NBTTL mechanism, we need to find:

- The input-output relation between the crank OA angular rotation and the coupler point, $\mathrm{D}$, displacement;

- The needle jerk in point $F$.

In this section, the modeling of the NBTTL mechanism is presented. The angle $\theta_{4}$, the velocity $\bar{\theta}_{4}$ and the acceleration $\bar{\theta}_{4}$ describing the input of the machine are given by the driving motor.

The needle displacement, with respect to (OX, OY) frame, is given by (Fig. 1):

$$
S=r_{7} \cos \left(\theta_{4}-\alpha\right)+r_{8} \cos \left(\sin ^{-1}\left(\frac{r_{7}}{r_{8}} \sin \left(\theta_{4}-\alpha\right)\right)\right)
$$

By taking the time derivative of equation (1), we can find the needle jerk:

$$
\begin{aligned}
& \bar{S}=-r_{7}\left[\bar{\theta}_{4} \sin \left(\theta_{4}-\alpha\right)+3 \bar{\theta}_{4} \bar{\theta}_{4} \cos \left(\theta_{4}-\alpha\right)-\bar{\theta}_{4}{ }^{3} \sin \left(\theta_{4}-\alpha\right)\right] \\
&- {\left[3 r_{7}\left[\frac{r_{7}}{r_{8}} \frac{d f\left(\theta_{4}\right)}{\mathrm{d} t} \bar{\theta}_{4}+\frac{r_{7}}{r_{8}} f\left(\theta_{4}\right) \bar{\theta}_{4}\right]\right.} \\
&\left.f\left(\theta_{4}\right) \bar{\theta}_{4} \cos \left(\sin ^{-1}\left(\frac{r_{7}}{r_{8}} \sin \left(\theta_{4}-\alpha\right)\right)\right)\right] \\
&-\left[r_{7} \sin \left(\theta_{4}-\alpha\right) \frac{d}{\mathrm{~d} t}\left[\frac{r_{7}}{r_{8}} \frac{d f\left(\theta_{4}\right)}{\mathrm{d} t} \bar{\theta}_{4}+\frac{r_{7}}{r_{8}} f\left(\theta_{4}\right) \bar{\theta}_{4}\right]\right] \\
&+\left(\frac{r_{7}}{r_{7}} f\left(\theta_{4}\right) \bar{\theta}_{4}\right)^{3} r_{7} \sin \left(\theta_{4}-\alpha\right)
\end{aligned}
$$

where $f\left(\theta_{4}\right)=\frac{\cos \left(\theta_{4}-\alpha\right)}{\sqrt{1-\left(\frac{r_{7}}{r_{8}}\right)^{2} \sin ^{2}\left(\theta_{4}-\alpha\right)}} .(3)$ 


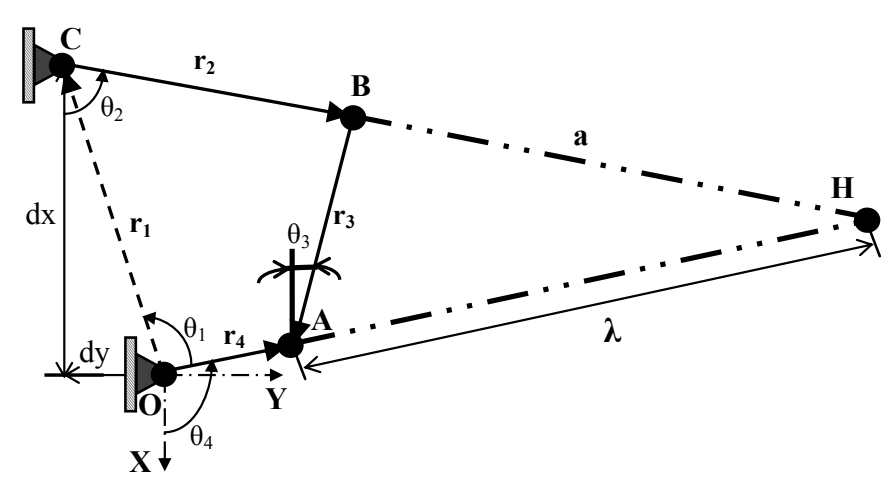

Fig. 2. Position of a virtual loop of a four-bar linkage.

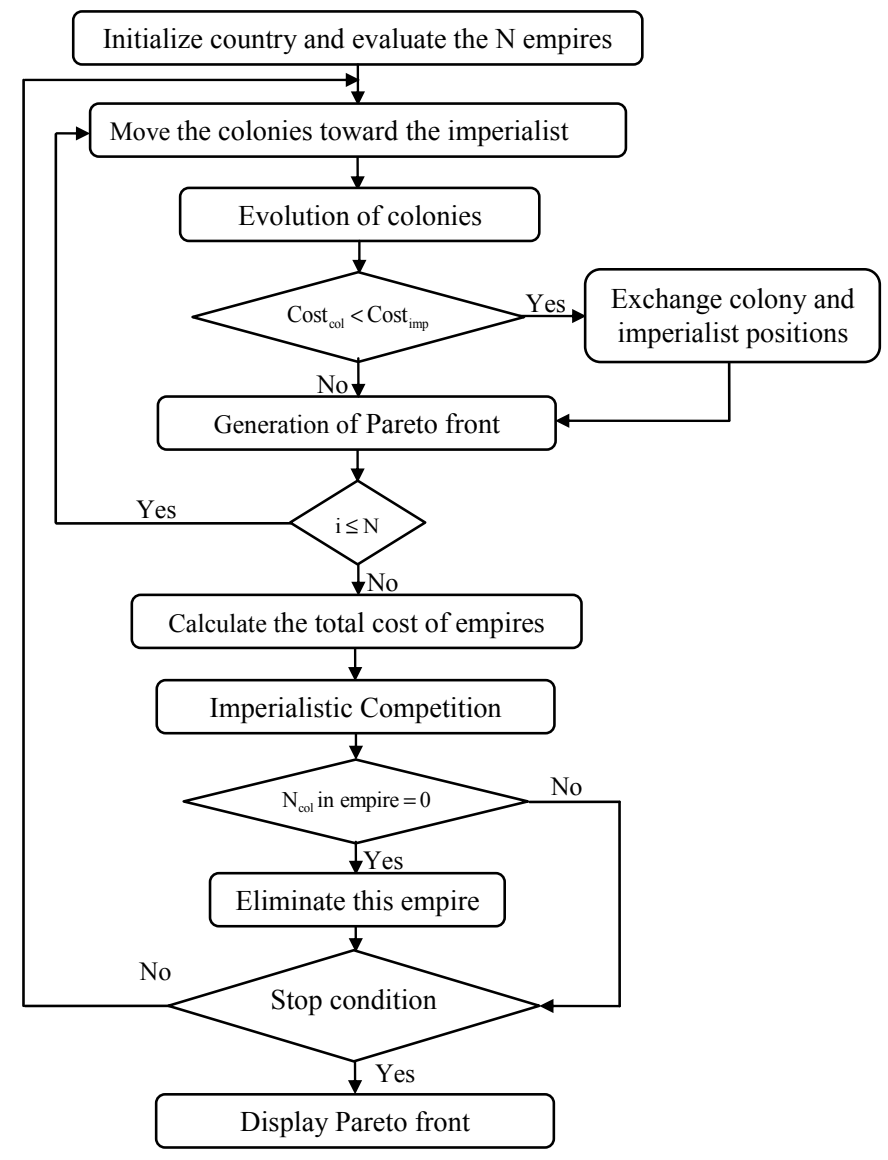

Fig. 3. The MOICA flowchart.

In textile engineering, sewing machines have been basically designed for work at high speed. This can lead to high vibration. To decrease the vibration of the sewing machine, it is recommended to reduce the jerk of its needle given by:

$$
\mathrm{NJ}=\int_{0}^{2 \pi}|\bar{S}| d \theta_{4}
$$

NJ defines the first objective function to be minimized.
Generally, the maximum angular velocity ratio (MAVR) of the mechanism is treated geometrically [12]. In this work we will give a closed form solution of the MAVR. Referring to Figure 2, $\mathrm{H}$ is the intersection of the two lines (CB) and (OA). It represents the instantaneous rotation centre of link $r_{3}$ with respect to link $r_{1}$. The closed loop equations of the virtual loop $\mathrm{ABH}$ can be given by:

$$
\overrightarrow{\mathrm{AB}}+\overrightarrow{\mathrm{BH}}+\overrightarrow{\mathrm{HA}}=\overrightarrow{0}
$$

Equation (5) can be projected into their $\mathrm{X}$ and $\mathrm{Y}$ components.

$$
\left\{\begin{array}{l}
-r_{3} \cos \theta_{3}+a \cos \theta_{2}-\lambda \cos \theta_{4}=0 \\
r_{3} \sin \theta_{3}+a \sin \theta_{2}-\lambda \sin \theta_{4}=0
\end{array}\right.
$$

By using equation (6), we can express $\lambda$ as follows:

$$
\lambda=r_{3} \frac{\sin \left(\theta_{3}+\theta_{2}\right)}{\sin \left(\theta_{4}-\theta_{2}\right)}
$$

The MAVR is given by:

$$
\text { MAVR }=\max (A V R)=\max \left(\frac{\bar{\theta}_{3}}{\bar{\theta}_{4}}\right)=\max \left(\frac{r_{4}}{\lambda}\right)
$$

From equations (7) and (8), the MAVR is given by:

$$
\operatorname{MAVR}=\max \left(\frac{r_{4} \sin \left(\theta_{4}-\theta_{2}\right)}{r_{3} \sin \left(\theta_{3}+\theta_{2}\right)}\right)
$$

Minimizing the MAVR yields the minimization of the input to output torque ratio. Minimizing this ratio allows the minimization of accelerations and hence minimizing the inertial forces of the mechanism. Hence, The MAVR represents the second objective function.

\section{Deterministic multi-objective optimization design of the NBTTL mechanism}

The multi-objective optimization problem can be defined as minimizing simultaneously the NJ and the MAVR under some constraints. In order to ensure that the input crank of the thread take-up lever can make a complete rotation, the mechanism should satisfy the Grashof law. Moreover, the set of input link angles $\theta_{4}$ has to vary in an increasing order $\left(\theta_{4}(i)<\theta_{4}(i+1)\right)$. The displacement of the needle, determined experimentally, is limited $(30 \mathrm{~mm} \leq S \leq 62 \mathrm{~mm})$ and the design variables have to be limited to some bounds due to the practical limitations in the construction of the mechanism. Hence, the multi-objective optimization problem can be formulated as:

$$
\operatorname{Minimize}\left\{\begin{array}{l}
\mathrm{NJ}=\int_{0}^{2 \pi}|\bar{S}| d \theta_{4} \\
\mathrm{MAVR}=\max \left(\frac{r_{4} \sin \left(\theta_{4}-\theta_{2}\right)}{r_{3} \sin \left(\theta_{3}+\theta_{2}\right)}\right)
\end{array} .\right.
$$




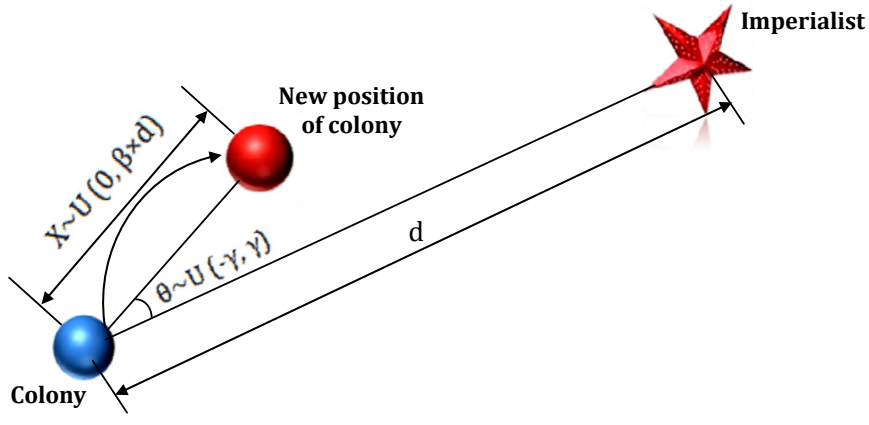

Fig. 4. Moving colonies toward their relevant imperialist.

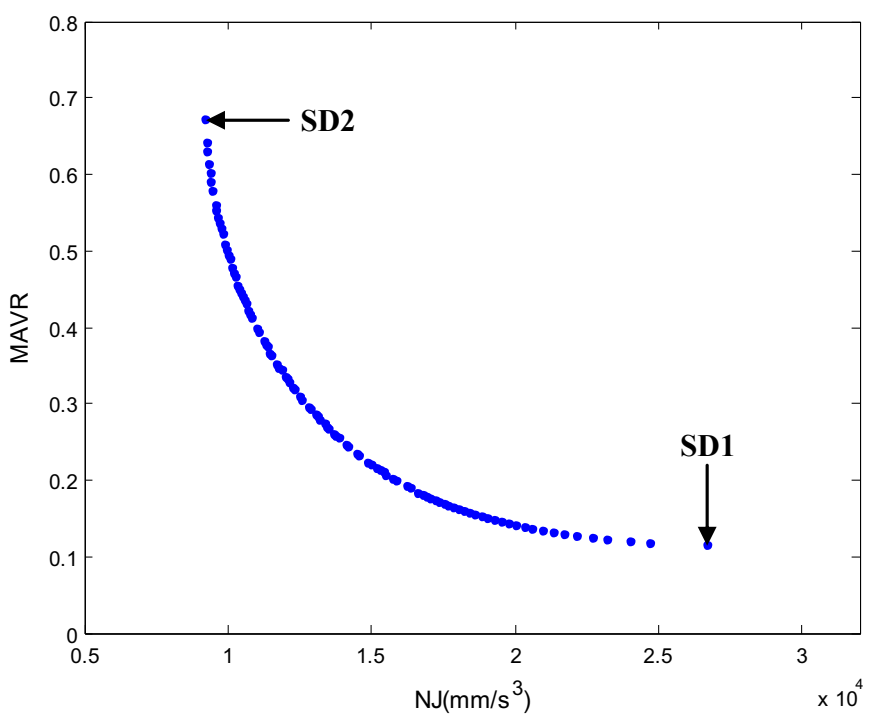

Fig. 6. The deterministic optimal solutions.

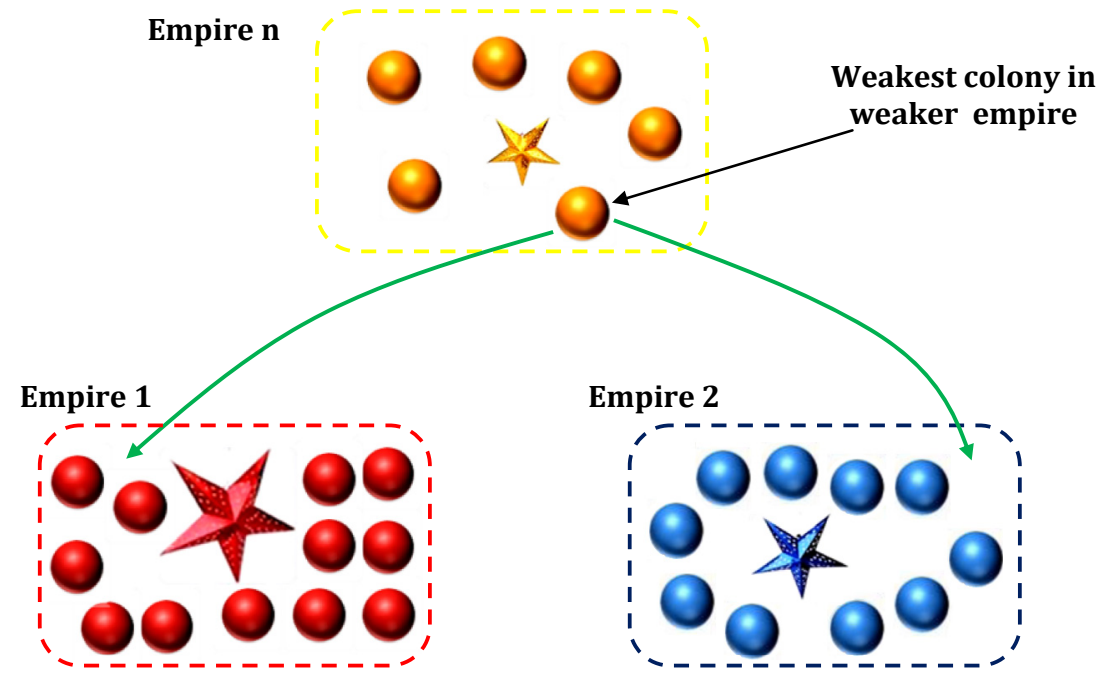

Fig. 5. Imperialistic competition.

Subject to : $\left\{\begin{array}{l}2\left[\max \left(r_{1}, r_{2}, r_{3}, r_{4}\right)+\min \left(r_{1}, r_{2}, r_{3}, r_{4}\right)\right] \\ <r_{1}+r_{2}+r_{3}+r_{4} \\ \theta_{4}^{i}-\theta_{4}^{i+1}<0 \\ \sin \left(\theta_{3}+\theta_{2}\right) \neq 0 \\ \operatorname{DP} \in \mathrm{D}(\mathrm{DP}) \\ 30 \mathrm{~mm} \leq S \leq 62 \mathrm{~mm}\end{array}\right.$.

The search domain $\mathrm{D}(\mathrm{DP})$ of each $\mathrm{DP}$ is listed in Table 1.

To solve this optimization problem, the MOICA method [13] will be used.

\subsection{The optimization method}

The MOICA is a stochastic optimization method developed in [13]. This method is based on the ICA algorithm [13-15]. Figure 3 shows the diagram of the MOICA.
The MOICA begins with a generation of a random initial population. Each element of the population is a country. The country cost is fixed based on the evaluation of the objective functions. The "imperialist" is the most powerful country and the "colonies" are the other countries. The initial empires, composed by several colonies and one imperialist, are formed by the initial population. Each colony starts moving towards the more powerful imperialist (Fig. 4). In this movement, $\theta$ and $\mathrm{X}$ are numbers generated uniformly $X \sim U(0, \beta \times d)$ and $\theta \sim \mathrm{U}(-\gamma, \gamma)$. $d$ is the distance between the imperialist and the colony and $\beta$ must be greater than 1. $\gamma$ is a parameter representing the direction deviation [13-15].

In each empire, the random mutation and the crossover operators can improve the colonies quality by adopting more powerful new ones. Therefore, the powerful colony can permute positions with the imperialist. Based on the fast non-dominated sorting approach [16], the nondominated imperialists are stored in the Pareto front. 
Table 1. Range of the design variables.

\begin{tabular}{|c|c|c|c|c|}
\hline $\mathrm{DP}$ & $\mathrm{d} x(\mathrm{~mm})$ & $\mathrm{d} y(\mathrm{~mm})$ & $r_{i}(\mathrm{~mm})$ & $\alpha(\operatorname{deg})$ \\
\hline $\mathrm{D}(\mathrm{DP})$ & {$\left[\begin{array}{ll}0 & 60\end{array}\right]$} & {$\left[\begin{array}{ll}0 & 60\end{array}\right]$} & {$\left[\begin{array}{ll}0 & 60\end{array}\right]$} & {$\left[\begin{array}{ll}0 & 360\end{array}\right]$} \\
\hline
\end{tabular}

Table 2. The MOICA parameters.

Number of countries Number of imperialists Assimilation coefficient $\beta$ Crossover probability Mutation probability

\begin{tabular}{lllll}
\hline 1000 & 100 & 1.2 & 0.9 & 0.1 \\
\hline
\end{tabular}

Table 3. Design vector of SD1, SD2 and Juki 8700.

\begin{tabular}{llll}
\hline Parameters & SD1 & SD2 & Juki 8700 \\
\hline $\mathrm{d} x(\mathrm{~mm})$ & 28.64 & 36.17 & 38.64 \\
$\mathrm{~d} y(\mathrm{~mm})$ & 38.12 & 29.42 & 33.22 \\
$r_{2}(\mathrm{~mm})$ & 37.76 & 43.89 & 44.80 \\
$r_{3}(\mathrm{~mm})$ & 33.07 & 26.81 & 30.31 \\
$\mathrm{r}_{4}(\mathrm{~mm})$ & 7.06 & 8.42 & 7.68 \\
$r_{7}(\mathrm{~mm})$ & 12.63 & 13.04 & 11.57 \\
$r_{8}(\mathrm{~mm})$ & 44.26 & 47.79 & 49.82 \\
$\left(^{\circ}\right)$ & 19.76 & 16.81 & 18 \\
MAVR & 0.12 & 0.66 & 0.73 \\
NJ $\left(\mathrm{mm} \mathrm{s}^{-3}\right)$ & $2.67 \mathrm{E}+4$ & $9.25 \mathrm{E}+3$ & $2.81 \mathrm{E}+4$ \\
\hline
\end{tabular}

The sum of the imperialist power with a mean power proportion of its colonies, gives the empire power. During the imperialists competition all empires, based on their power, try the possession of the other empires colonies (Fig. 5). The weak empires lose their colonies and collapse. The MOICA algorithm stops when all the weak empires collapse except the powerful one. The MOICA parameters are given in Table 2.

\subsection{Results and discussions}

Using the MOICA algorithm, the Pareto front (Fig. 6) can be determined to find the optimal non-dominated solutions of the multi-objective optimization problem defined by equation (10) under the constraint presented by equation (11).

In Figure 6, each non-dominated solution represents an optimal design vector of the NBTTL mechanism. One can note that the NBTTL having the best MAVR, has the worst NJ and vice versa. Table 3 presents the design vectors of the extreme deterministic optimal solutions SD1 and SD2 taken from the Pareto front. For comparison reason, we give in the same table the design vector of the known sewing machine Juki 8700 [17].

Figure 7 shows the variation of the angular velocity ratio (AVR), for the extreme determinist optimal solutions SD1 and SD2, as a function of the input link angle $\theta_{4}$. For the sake of comparison, we present in the same figure the variation of the AVR obtained by the sewing machine Juki 8700 .
From Figure 7 one can note that, compared to commercialized sewing machine Juki 8700 , we can reduce the MAVR by about $80 \%$ by using the optimal SD1 solution. However, if we consider SD2 that minimizes the NJ we have a reduction of the MAVR by $9.5 \%$.

Figure 8 shows the evolution of the needle Jerk for SD1 and SD2 solutions and Juki 8700 . One can note that by considering the SD2 design vector, we decrease the needle jerk down by $70 \%$ compared to the one of the Juki 8700 .

The obtained results show that the MAVR and the Needle Jerk can be reduced by $80 \%$ and $70 \%$, respectively, in the case of the optimized mechanism, compared to Juki 8700 machine. However, in practice the DPs are not known precisely and can present uncertainties around their nominal values. These uncertainties can generate fluctuations of the NBTTL performances evaluated by NJ and MAVR. Therefore, it's interesting to find a robust optimal NBTTL mechanism where the performances have low sensitivity to the DP uncertainties.

\subsection{The NBTTL performance sensitivity}

In this section, we investigate the effect of the DP uncertainties on the NBTTL performance. For this goal, each DP is modeled by a normal distribution given by a mean value and a standard deviation. The MCS is performed for every DP where each evaluation consists of a specified number of runs (Fig. 9). For each MCS, all the deterministic design variables are fixed at their nominal 


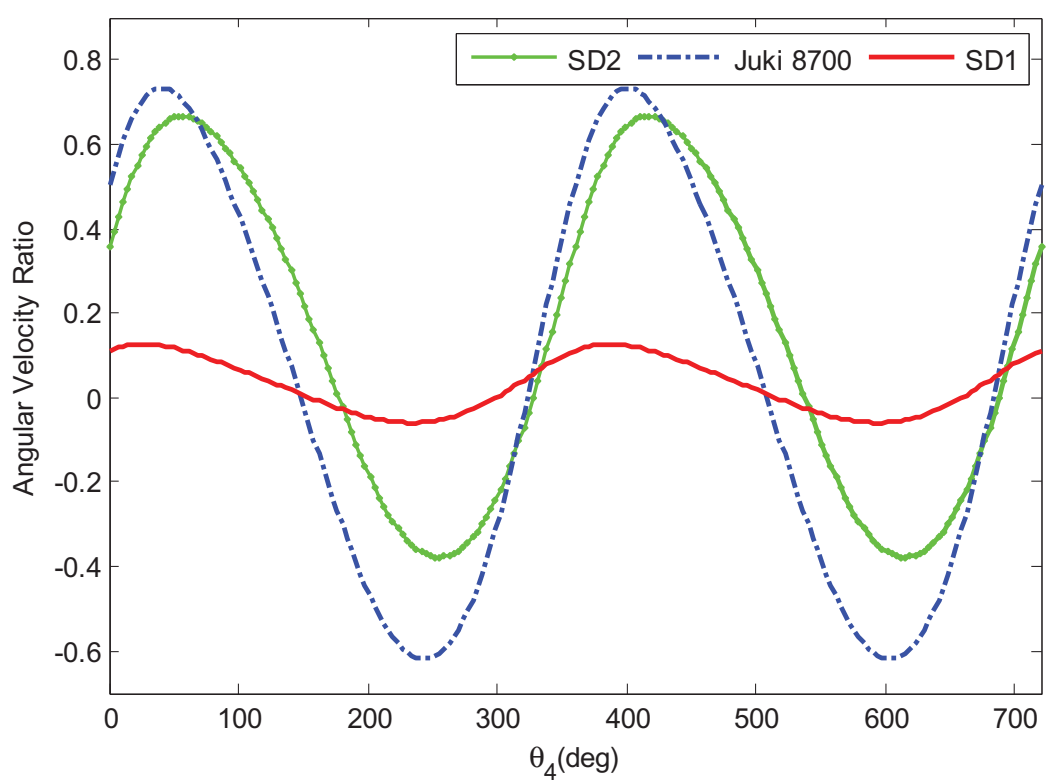

Fig. 7. Variation of the Angular Velocity Ratio for the three cases presented in Table 3.

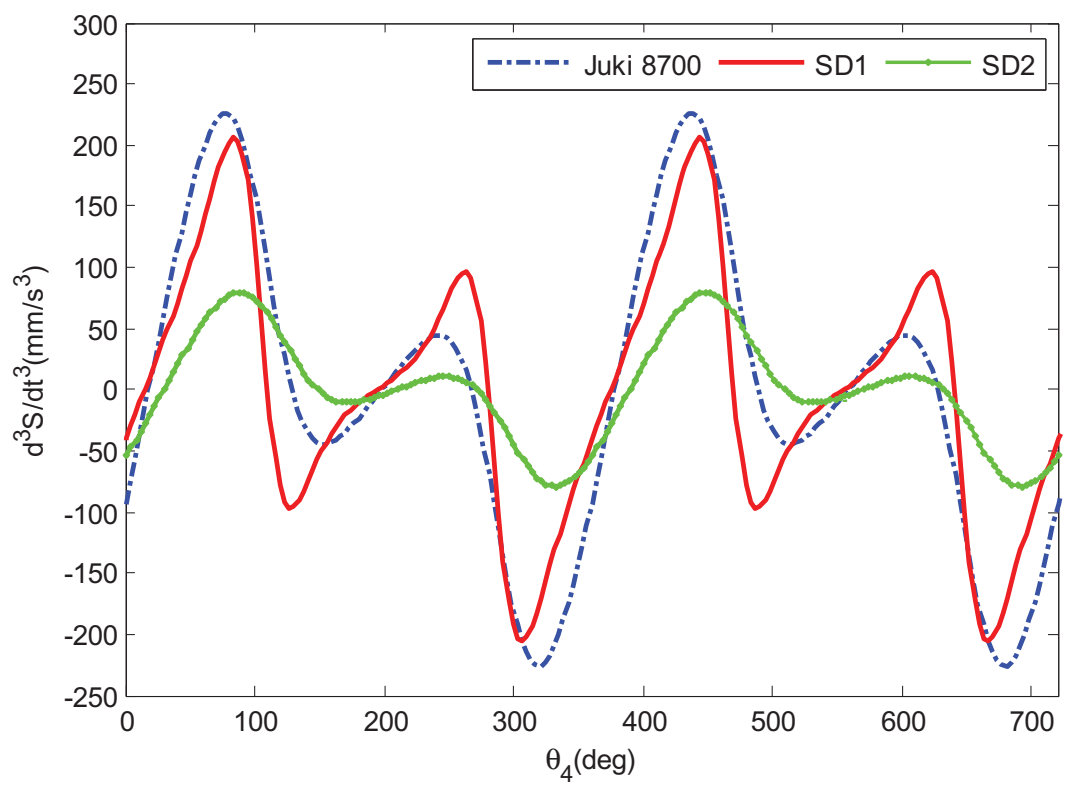

Fig. 8. Variation of the needle jerk for the three cases presented in Table 3.

values. The uncertainties are selected randomly from their statistical distributions supposed to vary within $\pm 10 \%$ of the DPs nominal values $[18,19]$.

With the MCS, we perform $n=10^{4}$ simulations to determine the mean values of the mechanism performances ( $\overline{\mathrm{NJ}}, \overline{\mathrm{MAVR}})$ and their respective standard deviations $\sigma_{\mathrm{NJ}}, \sigma_{\mathrm{MAVR}}$.

The performances of the obtained deterministic solutions are compared to those of the Juki 8700 machine. The determinist and Juki performances vulnerability to the DPs uncertainties are determined by introducing their corresponding DP under the MCS.

Figures 10 and 11 show the sensitivity of the NBTTL performances (MAVR and NJ) to the design parameters uncertainties for the extreme deterministic optimal solutions (SD1 and SD2). The variations of the same NBTTL performances, obtained by the sewing machine Juki 8700, are depicted in Figure 12.

Table 4 presents the mean and the sensitivity percentage of the NBTTL performances to the DP uncertainties for SD1, SD2 and Juki 8700. The sensitivity percentage of the NBTTL performance $u(u=$ NJ, MAVR) is evaluated as follows:

$$
S(\%)=100 \frac{3 \sigma_{u}}{\bar{u}}
$$

One can note that the performances of the deterministic optimal solutions (SD1 and SD2) are very sensitive to the DP uncertainties. In fact, a variation of $37 \%$ and $35 \%$ in the 


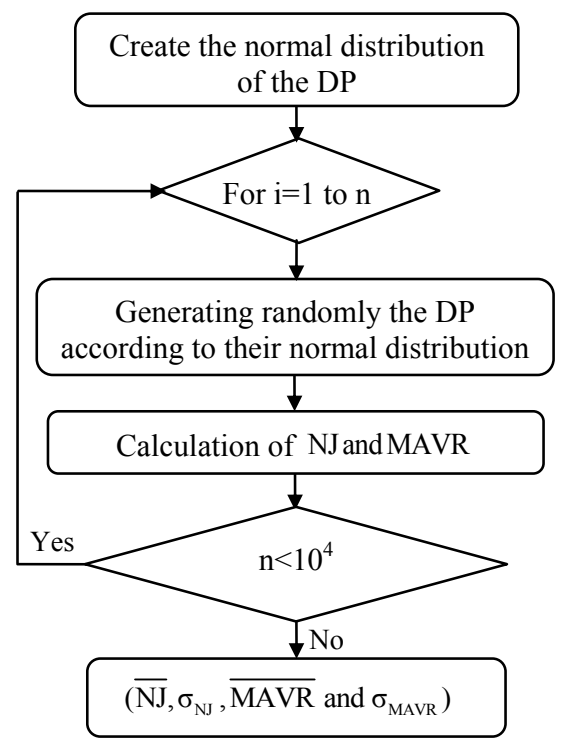

Fig. 9. The flowchart of MCS.
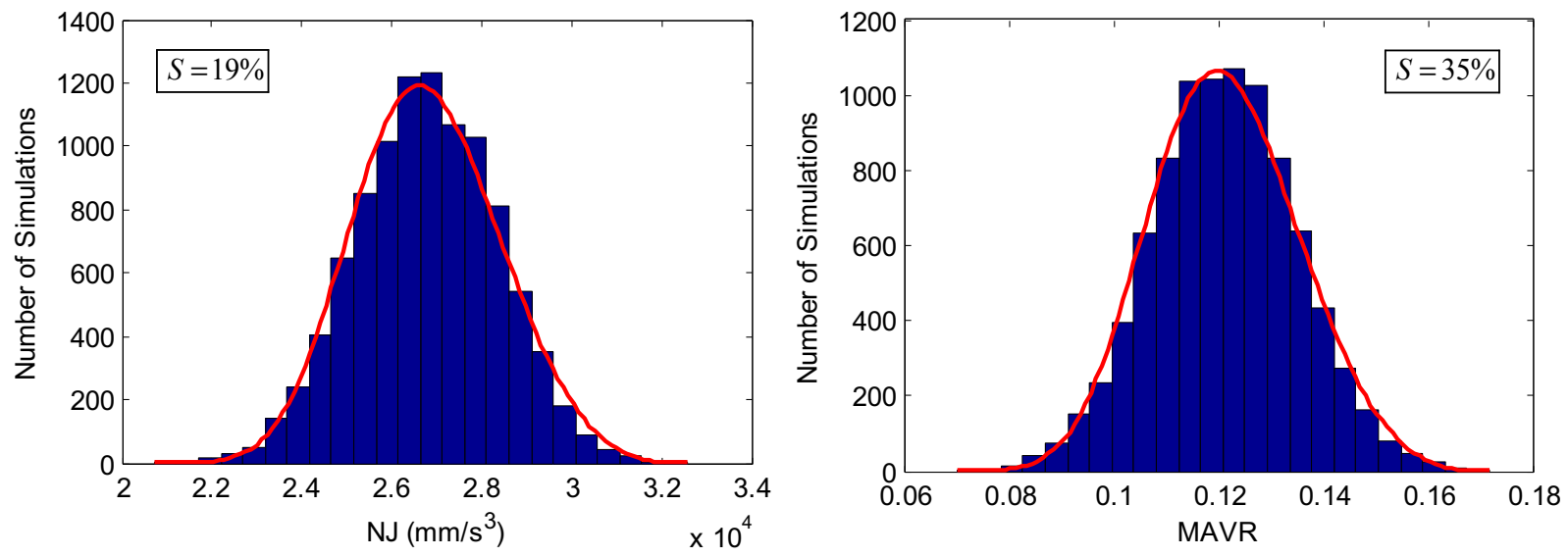

Fig. 10. Performance vulnerability of the solution SD1.

values of NJ and MAVR, respectively, are noted for uncertainties of $10 \%$ of the design parameters nominal values. Moreover, the Juki 8700 has a much lower NBTTL performances sensitivity compared with SD1 and SD2 solutions. The performances sensitivity of SD1 and SD2 solutions can reach the double of the Juki 8700 performances sensitivity. Therefore, a robust design of the NBTTL mechanism, that considers the mean value and the standard deviation of the machine performances, is necessary.

In what follows, we try to improve the deterministic optimization in order to obtain robust NBTTL performances to the DPs uncertainties. A multi-objective robust design, where the sensitivity of the NBTTL performances is considered as objective functions, will be conducted.

In order to have a robust design of the NBTTL mechanism, a combined algorithm that uses the MOICA and the MCS (MOICA-MCS) is developed and used.

\section{Robust design of the NBTTL mechanism}

A robust design of the NBTTL mechanism should have the minimum values of the objective functions NJ, MAVR and also these performances should have the minimum sensitivity generated by the DP uncertainty. This robustness can be quantified by the standard deviation of the objective function, i.e., high robustness of the solution means low standard deviation, for a given uncertainty of the DP. It is worth mentioning that all the solutions have to satisfy all the constraints imposed by the designer. Consequently the robust optimal design problem can be expressed as follows:

$$
\text { Minimize }\left\{\begin{array}{l}
\overline{\mathrm{NJ}} \\
\overline{\mathrm{MAVR}} \\
\sigma_{\mathrm{MAVR}} \\
\sigma_{\mathrm{NJ}}
\end{array}\right. \text {. }
$$



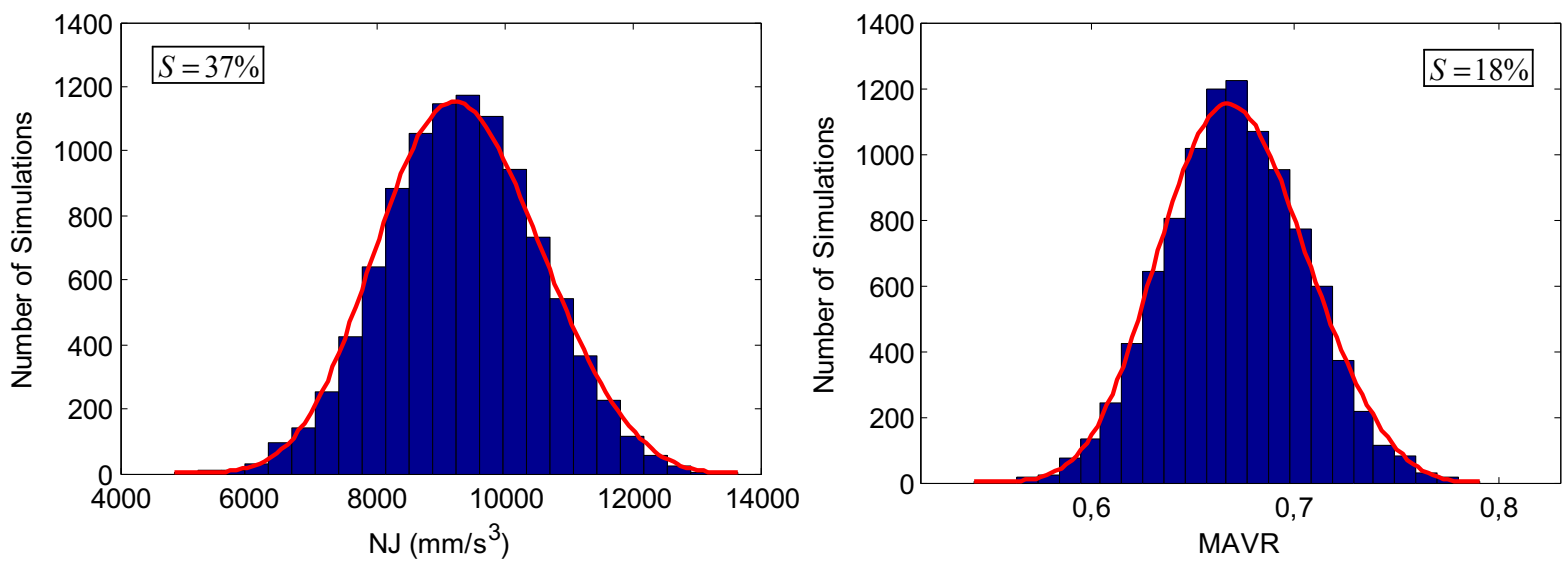

Fig. 11. Performance vulnerability of the solution SD2.
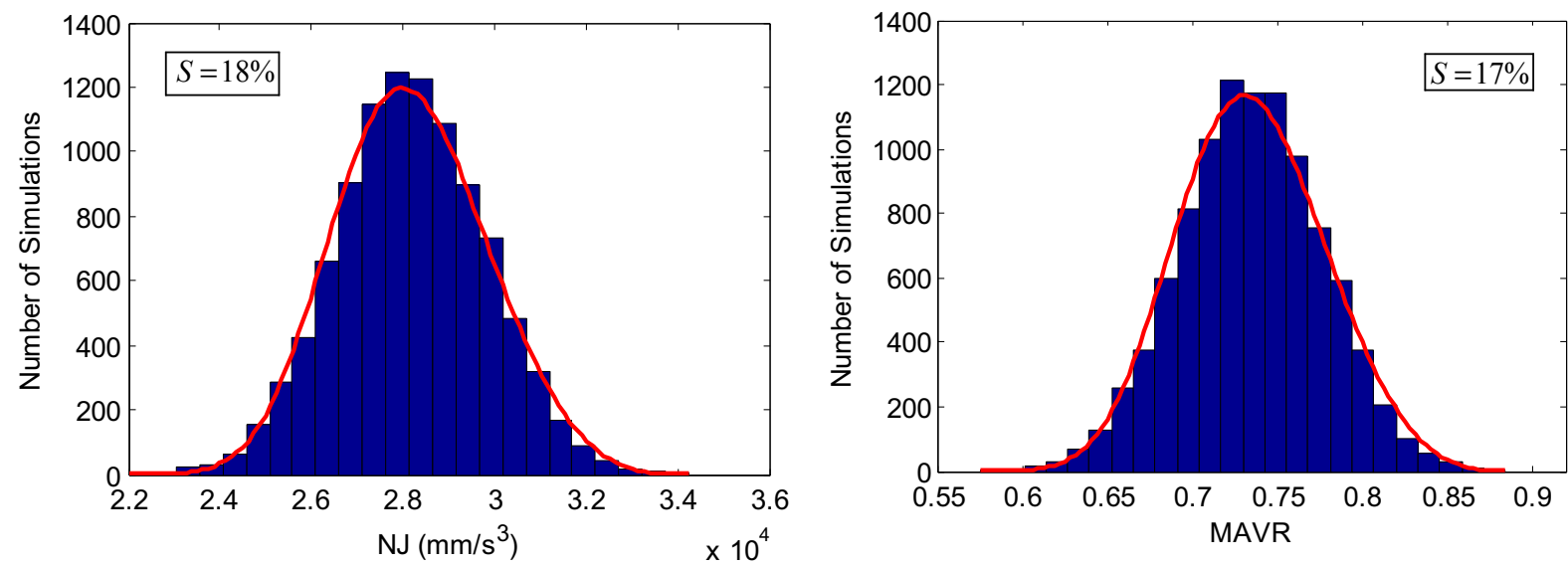

Fig. 12. Performance vulnerability of the Juki machine.

Table 4. Comparison of the performances results of SD1, SD2 and Juki 8700.

\begin{tabular}{|c|c|c|c|c|c|c|}
\hline \multirow[t]{2}{*}{ Solutions } & \multicolumn{2}{|c|}{ SD1 } & \multicolumn{2}{|c|}{ SD2 } & \multicolumn{2}{|c|}{ Juki 8700} \\
\hline & Mean value & $S(\%)$ & Mean value & $S(\%)$ & Mean value & $S(\%)$ \\
\hline $\mathrm{NJ}\left(\mathrm{mm} \mathrm{s}^{-3}\right)$ & $2.67 \mathrm{E}+4$ & 19 & $9.25 \mathrm{E}+3$ & 37 & $2.81 \mathrm{E}+4$ & 18 \\
\hline MAVR & 0.12 & 35 & 0.66 & 18 & 0.73 & 17 \\
\hline
\end{tabular}

Subject to : $\left\{\begin{array}{l}2\left[\max \left(\mathrm{r}_{1}, r_{2}, r_{3}, r_{4}\right)+\min \left(\mathrm{r}_{1}, r_{2}, r_{3}, r_{4}\right)\right] \\ <\mathrm{r}_{1}+r_{2}+r_{3}+r_{4} \\ \theta_{4}^{i}-\theta_{4}^{i+1}<0 \\ \sin \left(\theta_{3}+\theta_{2}\right) \neq 0 \\ \mathrm{DP} \in \mathrm{D}(\mathrm{DP}) \\ 30 \mathrm{~mm} \leq S \leq 62 \mathrm{~mm}\end{array}\right.$

\subsection{The MOICA-MCS algorithm}

In this section, the MOICA-MCS Algorithm (Fig. 13) that combines the MOICA and the MCS is developed and used to obtain robust optimal design of the NBTTNJ, MAVRL mechanisms. In fact, after the evolution stage, the MOICA sends a generation of countries to the MCS. The MCS performs $\mathrm{n}$ simulations for every country and sends back to the MOICA the mean values, $\overline{\mathrm{NJ}}$, and their standard deviations, $\sigma_{\mathrm{NJ}}, \sigma_{\mathrm{MAVR}}$. Then, these countries undergo the remaining steps of the MOICA algorithm. The MOICAMCS parameters are the same for MOICA (Tab. 2). The number of MCS $\mathrm{n}$ is fixed to $10^{4}$.

\subsection{Results and discussions}

The MOICA-MCS algorithm yields the non-dominated solutions presented in the Pareto front (Fig. 14). Each point represents a NBTTL mechanism design vector with the corresponding values of the four objective functions. 


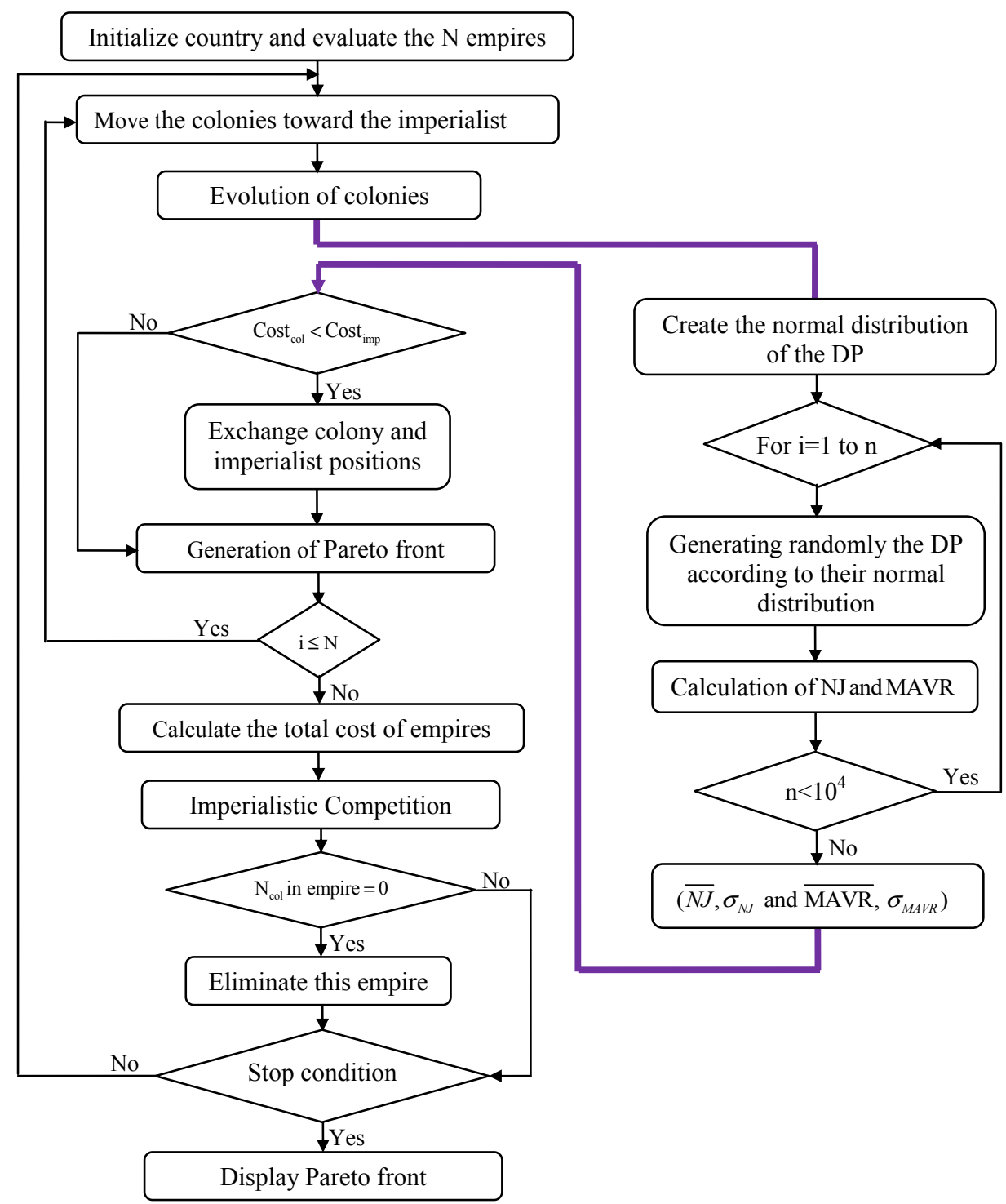

Fig. 13. The MOICA-MCS algorithm.

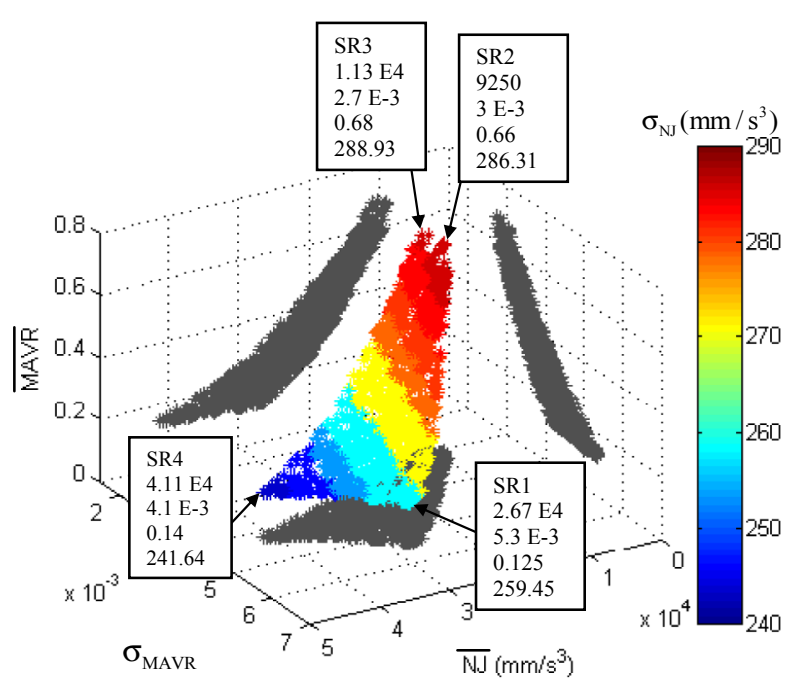

Fig. 14. The robust optimal solutions.
The solutions are more robust if the corresponding performance standard deviation is reduced. To represent graphically the four objective functions, different colors were used to represent the values of the fourth objective function, $\sigma_{\mathrm{NJ}}$.

The first remark is that $\overline{\mathrm{NJ}}$ and $\overline{\mathrm{MAVR}}$ tend to vary in the opposite direction of $\sigma_{\mathrm{NJ}}$ and $\sigma_{\mathrm{MAVR}}$, respectively. This means that reducing the average of an objective function leads inevitably to increasing its variability [20,21].

Table 5 presents the DPs of four extreme solutions from the Pareto front, as shown in Figure 14. By comparing these solutions, one can notice that the first solution presents the advantage of having the minimum of $\overline{\mathrm{MAVR}}$, the second one has the minimum of $\overline{\mathrm{NJ}}$, the third one has the minimum of $\sigma_{\mathrm{MAVR}}$ and the fourth one has the minimum of $\sigma_{\mathrm{NJ}}$.

To analyze the advantage of the robust optimal solutions compared with the deterministic ones, different sections of the robust Pareto front (given by Fig. 14), 
$\sigma_{N}\left(\mathrm{~mm} / \mathrm{s}^{3}\right)$

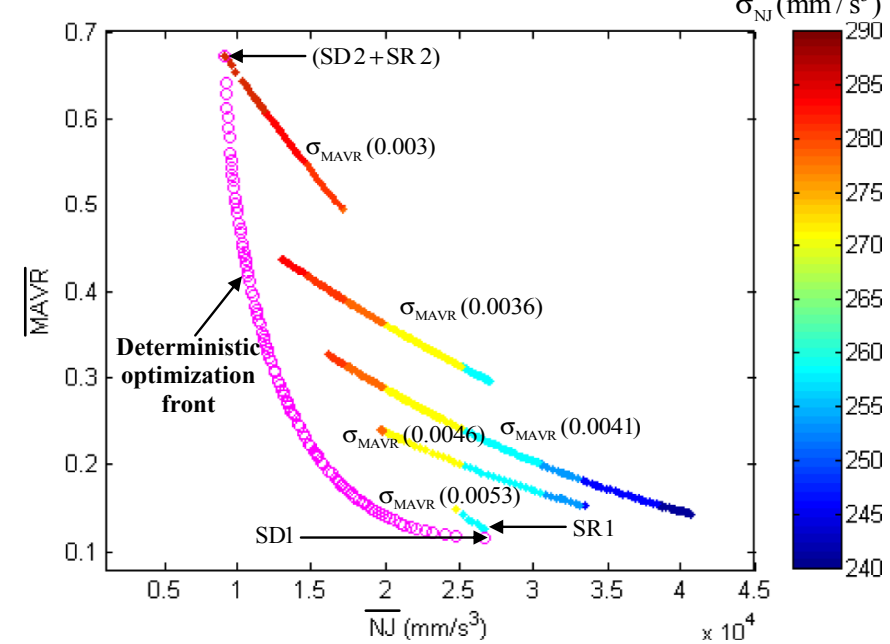

Fig. 15. Some configurations of robust solutions.

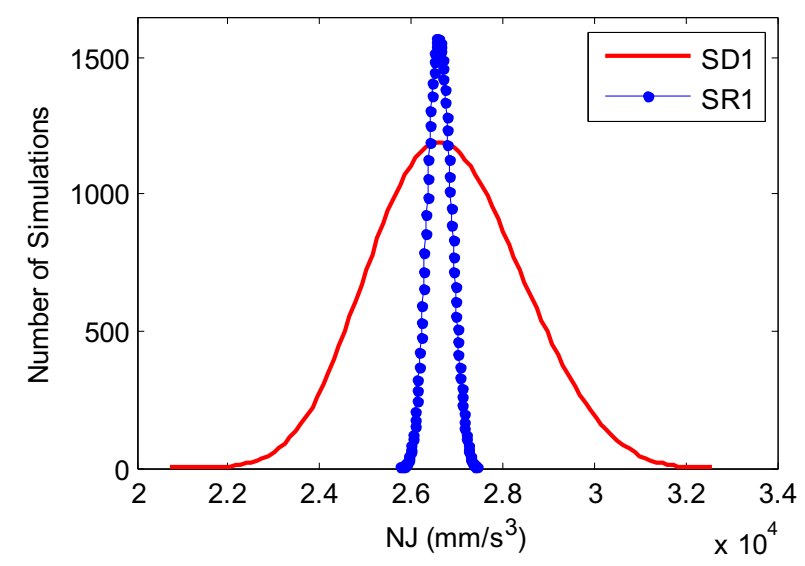

Table 5. Design vector of the extremely robust solutions.

\begin{tabular}{lllll}
\hline Parameters & SR1 & SR2 & SR3 & SR4 \\
\hline $\mathrm{d} x(\mathrm{~mm})$ & 37.64 & 34.16 & 29.71 & 32.47 \\
$\mathrm{~d} y(\mathrm{~mm})$ & 34.17 & 31.28 & 37.12 & 30.21 \\
$r_{2}(\mathrm{~mm})$ & 36.89 & 29.76 & 41.09 & 39.76 \\
$r_{3}(\mathrm{~mm})$ & 29.91 & 34.51 & 27.83 & 31.11 \\
$r_{4}(\mathrm{~mm})$ & 8.09 & 8.47 & 7.79 & 7.14 \\
$\mathrm{r}_{7}(\mathrm{~mm})$ & 11.74 & 12.49 & 11.91 & 12.87 \\
$r_{8}(\mathrm{~mm})$ & 43.82 & 44.86 & 45.39 & 44.28 \\
$\alpha\left(^{\circ}\right)$ & 15.86 & 17.64 & 19.67 & 21.07 \\
NJ $\left(\mathrm{mm} \mathrm{s}^{-3}\right)$ & $2.67 \mathrm{E}+4$ & 9250 & $1.13 \mathrm{E}+4$ & $4.11 \mathrm{E}+4$ \\
$\mathrm{MAVR}$ & 0.125 & 0.66 & 0.68 & 0.14 \\
$\sigma_{\mathrm{MAVR}}$ & $5.3 \mathrm{E}-3$ & $3 \mathrm{E}-3$ & $2.7 \mathrm{E}-3$ & $4.1 \mathrm{E}-3$ \\
$\sigma_{\mathrm{NJ}}$ & 259.45 & 286.31 & 288.93 & 242.64 \\
\hline
\end{tabular}

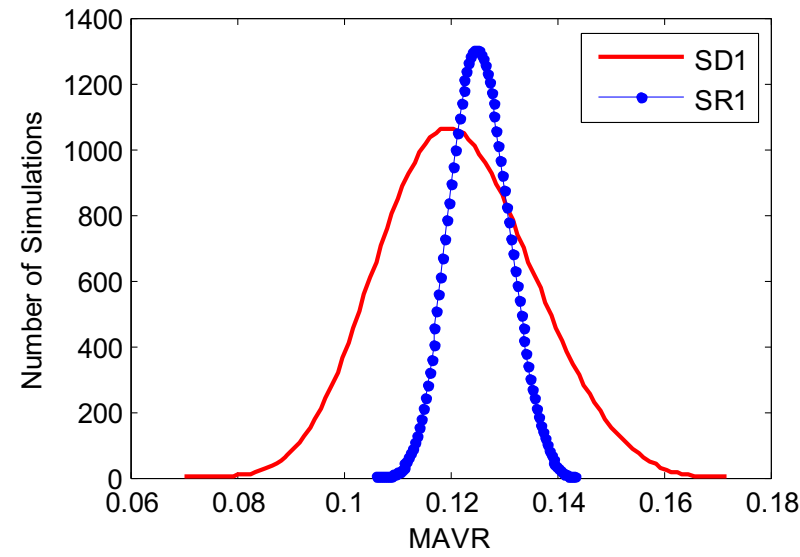

Fig. 16. Performance vulnerability of the solutions SD1 and SR1.
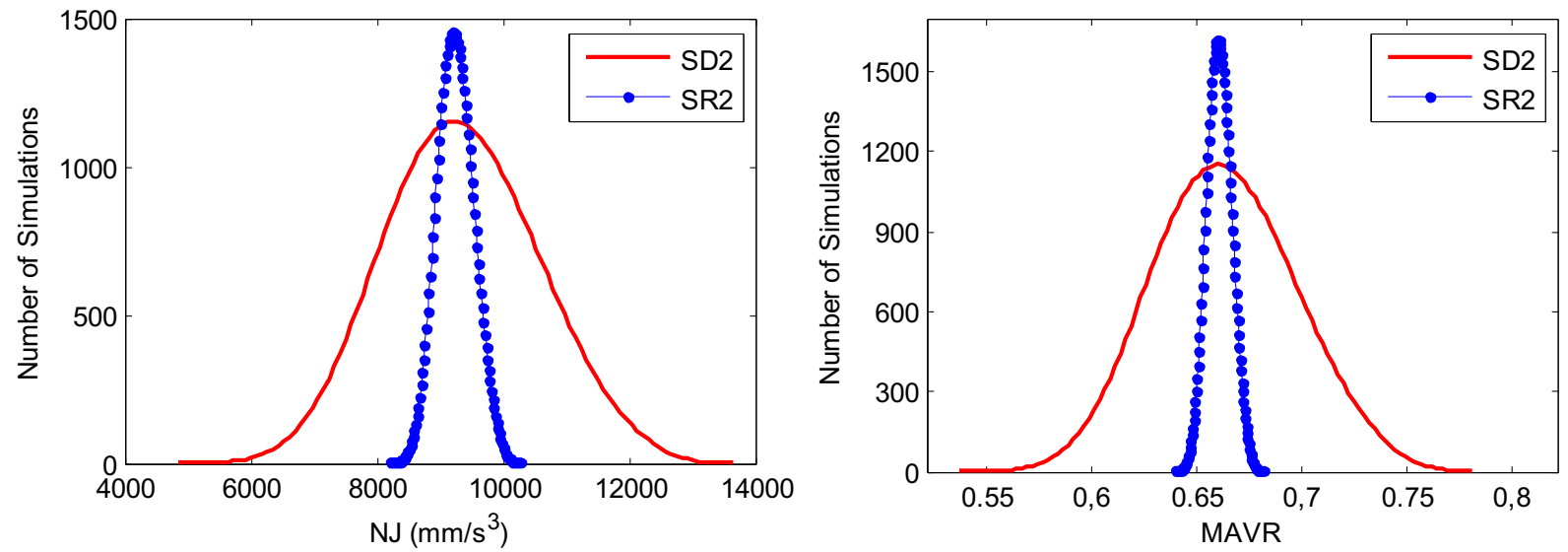

Fig. 17. Performance vulnerability of the solutions SD2 and SR2. 
Table 6. Performances comparison of SR1, SD1 and Juki one.

\begin{tabular}{|c|c|c|c|c|c|c|}
\hline \multirow[t]{2}{*}{ Solutions } & \multicolumn{2}{|c|}{ SR1 } & \multicolumn{2}{|c|}{ SD1 } & \multicolumn{2}{|c|}{ Juki 8700} \\
\hline & Mean value & $\mathrm{S}(\%)$ & Mean value & $\mathrm{S}(\%)$ & Mean value & $\mathrm{S}(\%)$ \\
\hline $\mathrm{NJ}\left(\mathrm{mm} / \mathrm{s}^{3}\right)$ & $2.67 \mathrm{E}+4$ & 3 & $2.67 \mathrm{E}+4$ & 19 & $2.81 \mathrm{E}+4$ & 18 \\
\hline
\end{tabular}

Table 7. Performances comparison of SR2, SD2 and Juki one.

\begin{tabular}{|c|c|c|c|c|c|c|}
\hline \multirow[t]{2}{*}{ Solutions } & \multicolumn{2}{|c|}{$\mathrm{SR} 2$} & \multicolumn{2}{|c|}{$\mathrm{SD} 2$} & \multicolumn{2}{|c|}{ Juki 8700} \\
\hline & Mean value & $\mathrm{S}(\%)$ & Mean value & $\mathrm{S}(\%)$ & Mean value & S (\%) \\
\hline $\mathrm{NJ}\left(\mathrm{mm} / \mathrm{s}^{3}\right)$ & 9250 & 9 & 9250 & 37 & $2.81 \mathrm{E}+4$ & 18 \\
\hline MAVR & 0.66 & 2 & 0.66 & 18 & 0.73 & 17 \\
\hline
\end{tabular}

corresponding to different values of $\sigma_{\mathrm{MAVR}}$, are presented in Figure 15. The deterministic Pareto front is also presented in the same figure.

For the comparison, the performance vulnerability of the robust solution (SR1) and the determinist one (SD1) are given in the same figure (Fig. 16). One can note that the SR1 solution has a comparable value of MAVR to the one obtained by the deterministic solution SD1. However, the performance of SR1 is much less sensitive to the DPs uncertainties (Tab. 6). Moreover, the robust solution SR1 has a slightly higher value for $\overline{\mathrm{MAVR}}(3 \%$ percentage difference), compared to the deterministic solution SD1, but with a much lower sensitivity to the DPs uncertainties.

Compared to the Juki 8700, one can note that SR1 has the lowest NJ with a sensitivity divided by about six (Tab. 6). Moreover, the value of $\overline{\mathrm{NJ}}$ and its sensitivity for SR1 are significantly improved.

From Figure 15, one can note also the coincidence of a robust and a deterministic solution (SR2 + SD2). The performance vulnerability of these two solutions are given in Figure 17 and Table 7. One can remark that for the same values for $\overline{\mathrm{MAVR}}$ and $\overline{\mathrm{NJ}}\left(\mathrm{mm} \mathrm{s}^{-3}\right)$ of the deterministic solution SD2, the robust solution SR2 presents a much lower variability to DPs uncertainties.

Compared to the Juki 8700 , one can note that SR2 solution is much more robust to the DPs uncertainties (Tab. 7).

One can conclude that finding a deterministic optimal solution can lead to solutions highly sensitive to the uncertainty of the design parameters. Therefore, the robust optimization strategy is recommended when seeking optimal solutions where the DPs are known with a certain uncertainty. This procedure was applied to the case of the NBTTL mechanism and we showed that the obtained solutions are highly robust to the DP uncertainties.

\section{Conclusion}

This paper deals with the multi-objective robust design optimization of the NBTTL mechanism. First, the mechanism is presented and analyzed to calculate the two objective functions, i.e., the jerk and the angular velocity ratio. Then, a deterministic optimization was conducted. It was shown that the optimal deterministic solutions are highly sensitive to the design parameter uncertainties. An algorithm, that combines the multiobjective imperialist competitive algorithm and the Monte Carlo Simulation, is developed and used for the multiobjective robust design optimization of the NBTTL mechanism. In the robust design, the NBTTL performances and their corresponding standard deviations are minimized, simultaneously.

The obtained results showed that the developed algorithm can yield robust designs of the sewing machine with similar performances as the those of the obtained by the deterministic optimization. However, with the robust design approach the performances of the obtained solutions are highly insensitive to the design parameter uncertainties.

\section{References}

[1] P. Payvandy, S. Ebrahimi, Optimization of the thread takeup lever mechanism in lockstitch sewing machine using the imperialistic competitive algorithm, J. Text. Polym. 3 (2015) $12-18$

[2] S. Ebrahimi, P. Payvandy, Optimization of the link drive mechanism in a sewing machine using imperialist competitive algorithm, Int. J. Cloth. Sci. Technol. 26 (2014) 247-260

[3] J. Kamerec, J. Beran, M. Lima, M. Machado, J.M. Machado, J.P. Silva, Finding the optimal setting of the sewing needle transfer mechanism using simulation software, in: TRS 2012 41st Textile Research Symposium, Portugal, 2012

[4] B. Peherstorfer, T. Cui, Y. Marzouk, K. Willcox, Multifidelity importance sampling, Comput. Methods Appl. Mech. Eng. 300 (2016) 490-509

[5] M. Chandrashekhar, R. Ganguli, Uncertainty handling in structural damage detection using fuzzy Logic and probabilistic simulation, Mech. Syst. Signal Process. 23 (2009) 384404

[6] S. Cloupet, L. Chevalier, H. Chollet, Probabilistic simulation of wear in loaded rolling contact, Mech. Ind. 8 (6) (2007) 609630

[7] M. Motevalli, A. Zadbar, E. Elyasi, M. Jalaal, Using MonteCarlo approach for analysis of quantitative and qualitative operation of reservoirs system with regard to the inflow uncertainty, J. Afr. Earth Sci. 105 (2015) 1-16

[8] S. Cheng, M. Li, Robust optimization using hybrid differential evolution and sequential quadratic programming, Eng. Optim. 47 (1) (2015) 87-106 
[9] M. Kalantari, C. Dong, Ian J. Davies, Multi-objective robust optimization of unidirectional carbon/glass fibre reinforced hybrid composites under flexural loading, Compos. Struct. 138 (2016) 264-275

[10] G. Sun, J. Fang, X. Tian, G. Li, Q. Li, Discrete robust optimization algorithm based on Taguchi method for structural crashworthiness design, Expert Syst. Appl. 42 (2015) 4482-4492

[11] S. Ghanmi, M. Guedri, M. L. Bouazizi, N. Bouhaddi, Robust multi-level and multi-objective optimization of complex mechanical structures, Mech. Ind. 11 (2010) $393-400$

[12] M. Khorshidi, M. Soheilypour, M. Peyro, A. Atai, M. Shariat Panahi, Optimal design of four-bar mechanisms using a hybrid multi-objective GA with adaptive local search, Mech. Mach. Theor. 46 (2011) 1453-1465

[13] B. Najlawi, M. Nejlaoui, Z. Affi, L. Romdhane, An improved imperialist competitive algorithm for multi-objective optimization, Eng. Optim. 48 (11) (2016) 1823-1844

[14] S. Hosseini, A. Al Khaled, A survey on the Imperialist competitive algorithm metaheuristic: Implementation in engineering domain and directions for future research, Appl. Soft Comput. 24 (2014) 1078-1094
[15] M.R. Ghaderi, M. Aghakhani, A. Eslampanah, K. Ghaderi, The application of imperialist competitive algorithm for optimization of deposition rate in submerged arc welding process using $\mathrm{TiO}_{2}$ nanoparticle, J. Mech. Sci. Technol. (2015) 357-364

[16] K. Deb, A. Pratap, S. Agarwal, T. Meyarivan, A fast and elitist multi-objective genetic algorithm: NSGA-II, IEEE Trans. Evol. Comput. 6 (2) (2002) 182-197

[17] B. Najlawi, M. Nejlaoui, Z. Affi, L. Romdhane, Multi-objective design optimization of the NBTTL Mechanism, in: International conference on acoustics and vibration, Tunisia, 2016

[18] M. Vallerio, D. Telen, L. Cabianca, F. Manenti, J.V. Impe, F. Logist, Robust multi-objective dynamic optimization of chemical processes using the Sigma Point method, Chem. Eng. Sci. 140 (2016) 201-216

[19] M. Martinez, S. Kotov, D. De Vleeschouwer, D. Pas, H. Pälike, Testing the impact of stratigraphic uncertainty on spectral analyses of sedimentary series, Clim. Past 12 (2016) 1765-1783

[20] G.C. Marano, S. Sgobba, R. Greco, M. Mezzina, Robust optimum design of tuned mass dampers devices in random vibrations mitigation, J. Sound Vib. 313 (2008) 472-492

[21] P. Nimmegeers, D. Telen, F. Logist, J. Van Impe, Dynamic optimization of biological networks under parametric uncertainty, BMC Syst. Biol. 10 (2016) 86

Cite this article as: M. Nejlaoui, B. Najlawi, Z. Affi, L. Romdhane, Multi-objective robust design optimization of the mechanism in a sewing machine, Mechanics \& Industry 18, 606 (2017) 